\title{
Formation and Photoluminescence of Fluorescent Polymers
}

\author{
Jinying Yuan, ${ }^{1}$ Shanfeng Wang, ${ }^{2}$ Jun Shan, ${ }^{3}$ Jing Peng, ${ }^{4}$ Liuhe Wei, ${ }^{5}$ and Xurong Xu ${ }^{6}$ \\ ${ }^{1}$ Department of Chemistry, Tsinghua University, Beijing 100084, China \\ ${ }^{2}$ Department of Materials Science and Engineering, The University of Tennessee, Knoxville, TN 37996, USA \\ ${ }^{3}$ KCL (Oy Keskuslaboratorio-Centrallaboratorium Ab), Tekniikantie, P.O. Box 70, 02151 Espoo, Finland \\ ${ }^{4}$ Department of Applied Chemistry, Peking University, Beijing 100871, China \\ ${ }^{5}$ Department of Chemistry, Zhengzhou University, Zhengzhou 450001, China \\ ${ }^{6}$ Departament of Chemistry, Zhejiang University, Hangzhou 310027, China
}

Correspondence should be addressed to Jinying Yuan, yuanjy@mail.tsinghua.edu.cn

Received 14 July 2010; Accepted 14 July 2010

Copyright (C) 2010 Jinying Yuan et al. This is an open access article distributed under the Creative Commons Attribution License, which permits unrestricted use, distribution, and reproduction in any medium, provided the original work is properly cited.

During the past several decades, there is an enormous demand for fluorescent materials, and research on fluorescent polymers has gathered great scientific attention because of their engrossing properties and important application in the fields of materials and life science. The latest research progress in fluorescent polymers is focused on the formation and photoluminescence of fluorescent polymers with new architecture. There are different methods to classify fluorescent polymers. For example, based on their solubility, fluorescent polymers can be divided into three types which are hydrophobic, hydrophilic and amphiphilic, individually. Various methods of design and synthesis of fluorescent polymers have also been developed. Fluorescent polymers can be synthesized by polymerization of fluorescent functional monomers, using fluorescent compounds as initiator, fluorescent compounds as chain transfer agents, chemical bonding between fluorescent groups and polymers, and polymerization of nonfluorescent functional monomer.

Fluorescent polymers are functional macromolecules with enormous important applications. Their emerging applications involve the fields of fluorescent probe, smart polymer machines, fluorescent chemosensor, fluorescent molecular thermometers, fluorescent imaging, drug delivery carriers, and so on. Polymers are convenient due to the fact that they are easily processable to small particles and thin films that can be deposited onto optical fibers and waveguides for sensor fabrication. Advanced strategies such as electrostatic layer-by-layer assembly and self-assembly of amphiphilic block copolymers containing chromophores have also been used for the formation of fluorescent systems.

The aim of this special issue is to bring forth the synergy between architecture and photoluminescence through new and significant contributions from active researchers in the field. All the papers in this issue are invited review or invited research papers by leading authorities and research groups in this field from universities, industry, and government laboratories.

The first paper of this special issue addresses the reversible addition-fragmentation chain transfer (RAFT) copolymerization of 2-((8-hydroxyquinolin-5-yl)methoxy)ethyl methacrylate (HQHEMA) with styrene (St) or methyl methacrylate (MMA) carried out in the presence of 2cyanoprop-2-yl dithionaphthalenoate (CPDN). The soluble polymers having tris (8-hydroxyquinoline)aluminum ( $\left.\mathrm{Alq}_{3}\right)$ and bis(8-hydroxyquinoline) $\mathrm{znic}(\mathrm{II})\left(\mathrm{Znq}_{2}\right)$ side chains were obtained via complexation of the polymers with aluminium isopropoxide or zinc acetate in the presence of monomeric 8-hydroxyquinoline, which had strong fluorescent emission at $520 \mathrm{~nm}$. The second paper is on the synthesis and characterization of new photoluminescent oxadiazole/carbazole-containing polymers (POCs). The polymers exhibit a high thermal stability, high glass transition temperatures, and good solubility in common organic solvents, despite the extended aromatic portion in the main polymer chain, and are potential candidate materials for fabricating blue light-emitting devices. The third paper proposes the synthesis and properties of a 
novel through-space conjugated polymer with a [2.2]paracyclophane skeleton. The obtained polymer possessed donor (fluorene) and acceptor (2,1,3-benzothiadiazole) segments that were alternately $\pi$-stacked in proximity via the [2.2] paracyclophane moieties.

The fourth paper presents the study on the printability of a copolyfluorene-fluorenone (PFFO) photoluminescent nanoparticle aqueous suspension on commercial tracing paper. This suspension was suited to inkjet or flexography printing techniques because it is composed of nanosized "pigments" (nanoparticles) and it showed suitable properties such as low viscosity and surface tension. The fifth paper describes that $\mathrm{H}$-aggregates of cyanine dye $\mathrm{Cy} 5$ covalently linked to the polymer PAH have been observed and the behavior of the "H-aggregate band" at different solvent and synthesis conditions has been investigated. In order to reduce the undesired $\mathrm{H}$-aggregates effect a different synthetic route in organic solvent has been developed yielding much higher label degrees and remarkable less $\mathrm{H}$-aggregates on the polymer. The final paper of this special issue is more forwardlooking. It addresses the technological applications of wormlike micelles and vesicles prepared from diblock copolymers. Although transmission electron microscopy has remained as the method of choice for assessing the morphologies, fluorescence microscopy has a number of advantages. When commercially available fluorophores are covalently attached to diblock copolymers, a number of their physicochemical characteristics can be investigated. This method becomes particularly useful for visualizing phase separation within polymer assemblies and assessing the dynamics of worm-like micelles in real time. Near-IR fluorophores can be covalently conjugated to polymers, and this opens the possibility for deep-tissue fluorescence imaging of polymer assemblies in drug delivery applications.

Jinying Yuan
Shanfeng Wang
Jun Shan
Jing Peng
Liuhe Wei
Xurong Xu



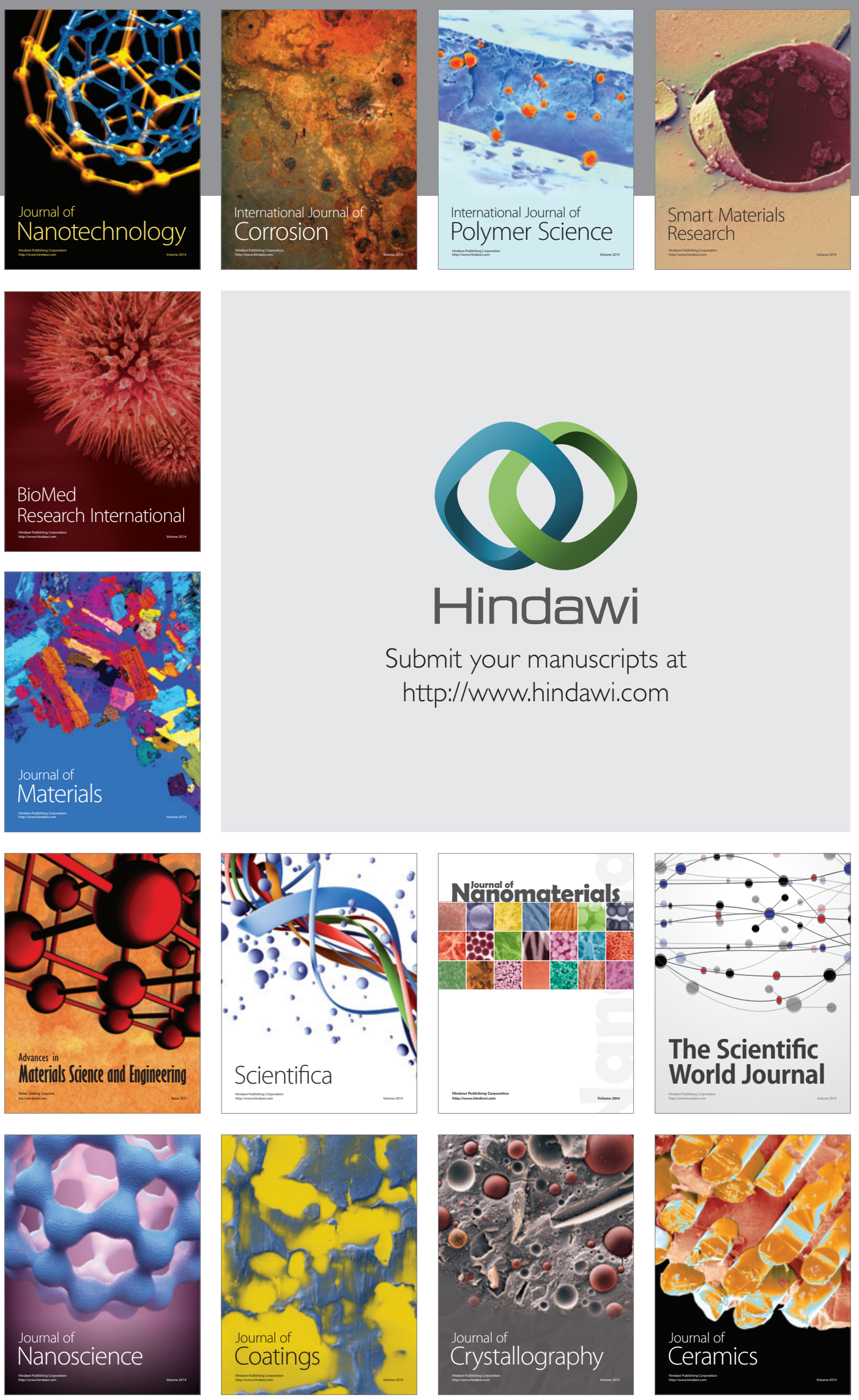

The Scientific World Journal

Submit your manuscripts at

http://www.hindawi.com

\section{World Journal}

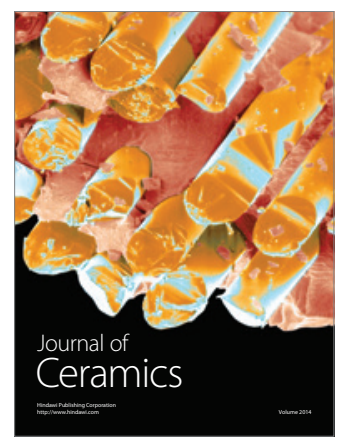

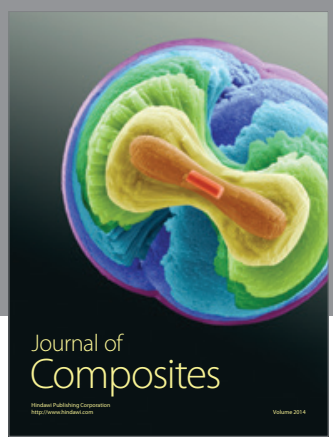
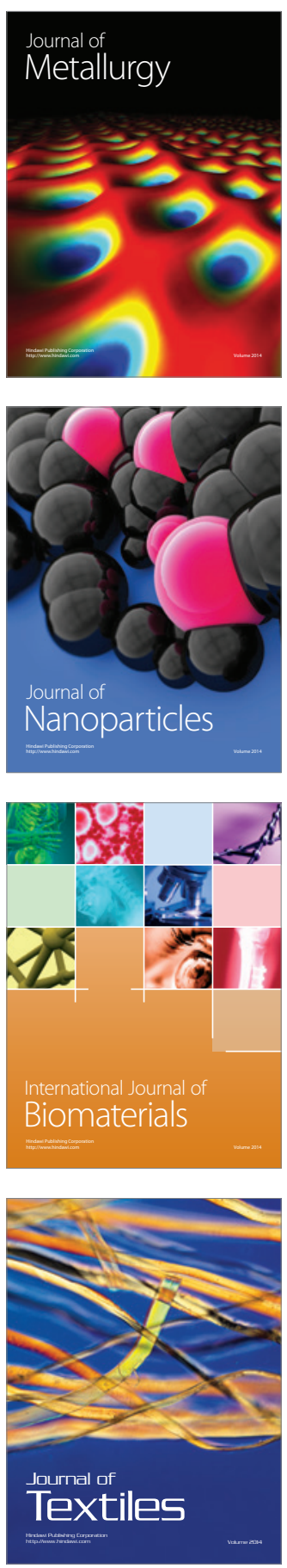